\title{
Die Legierungen von Mangan und Silber.
}

\author{
Von \\ G. Arrivant. ${ }^{1}$
}

Mit 2 Figuren im Text und 1 Tafel.

Die thermische Untersuchung der Legierungen von Mangan und Silber ist von G. Hindrichs ${ }^{2}$ ausgeführt worden, dessen Diagramm zeigt, daß sich die Bestandteile innerhalb der Konzentrationen von $30-90 \%$ Mangan in zwei Schichten sondern. Es wird auf teilweise Mischbarkeit in flüssigem Zustand und auf eine nicht merkliche Mischbarkeit im festen Zustande geschlossen.

Hindrichs hat, ebenso wie ich, zwischen 0 und $30 \%$ Mangan nur einen einzigen thermischen Effekt gefunden, der den Charakter eines Haltepunktes besitzt. Durch die Tatsache, daß er in Legierungen von 2-20\% Mangan einige manganreiche Tröpfchen fand, war er zu der Ansicht gekommen, daß das Fehlen der Mischbarkeit der beiden geschmolzenen Metalle sich bis etwa $2 \%$ Mangan erstrecke. Diese Meinung ist indessen nicht gerechtfertigt, wie sich aus dem folgenden ergibt, da der Mangel an Mischbarkeit sich nur bis etwa $30 \%$ Mangan erstreckt.

Da eine frühere Untersuchung dieser Frage auf anderen Wegen mich zu abweichenden Ergebnissen geführt hatte, ${ }^{3}$ hielt ich es für nützlich, diesen Gegenstand nochmals nach verschiedenen Methoden zu untersuchen, und zwar wählte ich einerseits die thermische Analyse, dann aber auch die mikroskopische Prüfung, die chemische Methode und die Bestimmung der elektromotorischen Kräfte der Aufiösung.

Als Ausgangsmaterialien dienten chemisch reines Silber und aluminothermisches Mangan, welches erhalten war durch Reduktion des reinen Oxyds mit pulverförmigem Aluminium in einem Magnesiatiegel unter Anwendung eines beträchtlichen Überschusses an

1 Aus dem Manuskript ins Deutsche übertragen von I. Koppex-Berlin.

2 Z. anorg. Chem. 59 (1908), 437.

${ }^{3}$ G. Arrivant, Procès verbaux des séances de la soc. des sc. phys. et nat. de Bordeaux 1903-04, S. 9.

Z. anorg. Chem. Bd. 88. Metallographisches Heft. 
Oxyd. Das Eisen, welches im allgemeinen durch das Aluminum eingeführt wird, war zum Teil vermieden durch die Benutzung von Feilspänen aus besonders reinen Metallbarren, die noch durch einen starken Elektromagneten gereinigt waren. ${ }^{1}$

In der Tat war der Eisengehalt des erhaltenen Mangans immer geringer als $0.5 \%$. Sein Schmelzpunkt wurde zu $1235^{\circ}$ gefunden, während Heraeus ${ }^{2} 1247^{\circ}$ und Hindrichs ${ }^{3} 1207^{\circ}$ (bezogen auf Smp. $\mathrm{Ni}=1451^{\circ}$ ) angaben. Die Temperaturen wurden bestimmt mit Hilfe eines Thermoelementes aus Platin und Platin-Rhodium, das geeicht worden war mit den Schmelzpunkten von Antimon $\left(630^{\circ}\right)$, von Silber $\left(961^{\circ}\right)$ und von Nickel $\left(1451^{\circ}\right)$, wobei man die üblichen Korrekturen anbrachte.

Die erhaltenen Resultate sind in der Tabelle 1 zusammengestellt, nach welcher das Diagramm gezeichnet ist.

(Siehe Tabelle 1, S. 195).

Der Zusatz einer kleinen Menge Silber zum Mangan erniedrigt dessen Schmelzpunkt bis auf $1180^{\circ}$. Die Konzentration des Punktes $D$ der festen Lösung von Silber-Mangan, welche an Silber gesättigt ist, beträgt ungefähr $94 \%$ Mangan. Hindricens hat für diesen

dewrichtsproz. Manzan

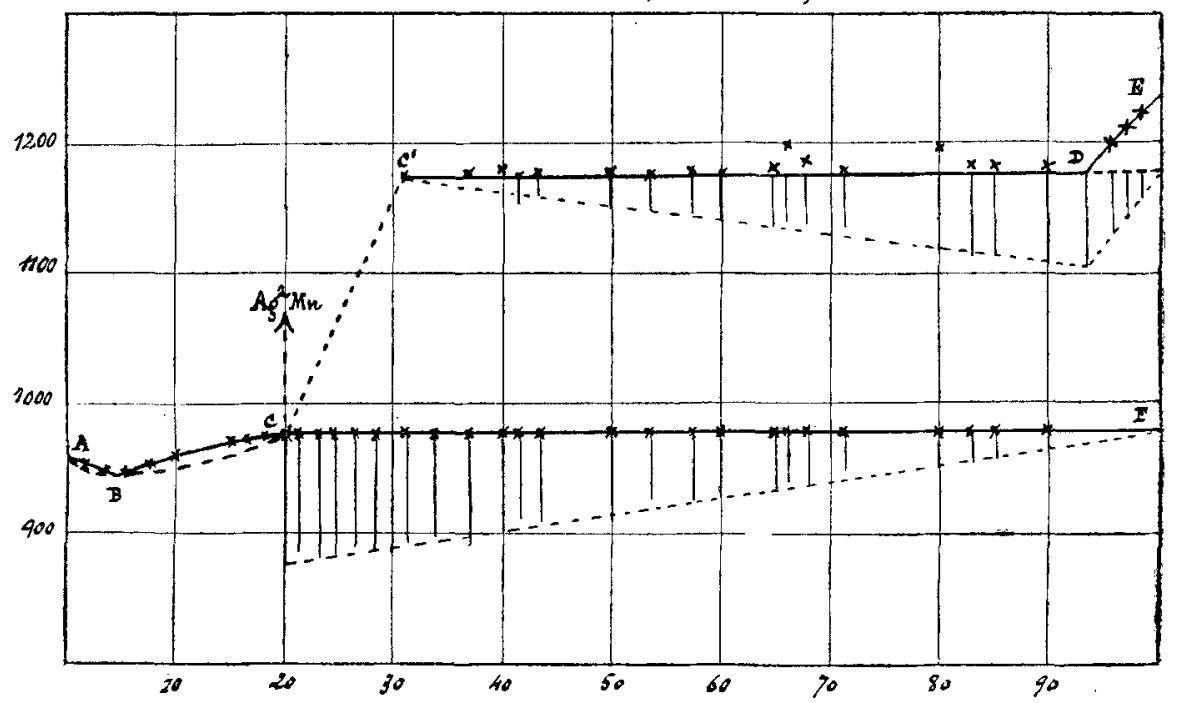

Fig. 1.

${ }^{1}$ G. Arrivant, C. R. soc. des sc. phys. et nat. de Bordeaux 4 (1908), 67.

${ }^{2}$ Zeitschr. f. Elektrochem. 8, $185 . \quad{ }^{3}$ Hindrichs, l. c. 
Tabelle 1.

\begin{tabular}{|c|c|c|c|c|c|c|}
\hline Nr. & $\begin{array}{c}\mathrm{Mn} \\
\text { in } \%\end{array}$ & $\begin{array}{c}\text { Gew. d. } \\
\text { Regulus in } \mathrm{g}\end{array}$ & $\begin{array}{l}\text { Temp. der } \\
\text { 1. Krist. in }{ }^{0}\end{array}$ & $\begin{array}{l}\text { Dauer } \\
\text { in Sek. }\end{array}$ & $\begin{array}{l}\text { Temp. der } \\
\text { 2. Krist. in }\end{array}$ & $\begin{array}{l}\text { Dauer } \\
\text { in Sek. }\end{array}$ \\
\hline & Ag rein & & & & 961 & \\
\hline 14 & 2.5 & 30 & & & 958 & \\
\hline 23 & 4 & 26 & & & 955 & \\
\hline 10 & 6 & 30 & & & 957 & \\
\hline 24 & 7.40 & 27 & & & 959 & \\
\hline 25 & 10.70 & 28 & & & 963 & \\
\hline 26 & 16.60 & 30 & & & 967 & \\
\hline 27 & 17.35 & 31 & & & 969 & \\
\hline 28 & 18.90 & 32 & & & 973 & \\
\hline 29 & 19.10 & 33 & & & 978 & \\
\hline 30 & 20.15 & 33 & & & 978 & 130 \\
\hline 31 & 21.50 & 34 & & & 980 & 135 \\
\hline 32 & 23.50 & 33 & & & 980 & 120 \\
\hline 6 & 25 & 30 & & & 978 & 80 \\
\hline 32 & 27 & 36 & & & 980 & 100 \\
\hline 33 & 29 & 38 & & & 975 & 100 \\
\hline 51 & 31 & 25 & 1170 & & 978 & 80 \\
\hline 52 & 34 & 38 & 1170 & & 978 & 90 \\
\hline 34 & 37.50 & 40 & 1180 & & 980 & 80 \\
\hline 5 & 40 & 27 & 1190 & 22 & 980 & 75 \\
\hline 47 & 41.15 & 23 & 1190 & 22 & 976 & 75 \\
\hline 35 & 43.50 & 21 & 1190 & 22 & 978 & 80 \\
\hline 36 & 50 & 23 & 1185 & 30 & 978 & 70 \\
\hline 4 & 54 & 31 & 1185 & 50 & 980 & 65 \\
\hline 37 & 58.50 & 30 & 1175 & 60 & 980 & 65 \\
\hline 38 & 60 & 25 & 1170 & 80 & 976 & 60 \\
\hline 45 & 65 & 30 & 1170 & 100 & 978 & 60 \\
\hline 3 & 66 & 27 & 1200 & 80 & 980 & 59 \\
\hline 39 & 68.50 & 30 & 1180 & 90 & 980 & 50 \\
\hline 2 & 71 & 34 & 1175 & 100 & 978 & 40 \\
\hline 44 & 80 & 29 & 1200 & 130 & 976 & 30 \\
\hline 43 & 82 & 22 & 1175 & 140 & 980 & 25 \\
\hline 46 & 85 & 21 & 1180 & 150 & 980 & 15 \\
\hline 1 & 90 & 30 & 1180 & 180 & 980 & 10 \\
\hline 42 & 95 & 23 & 1200 & 180 & 980 & \\
\hline 41 & 96 & 21 & 1210 & 180 & 980 & \\
\hline \multirow{2}{*}{50} & 97 & 30 & 1215 & 180 & 980 & \\
\hline & Mn rein & & 1235 & 180 & 980 & \\
\hline
\end{tabular}

Punkt $1148^{\circ}$ (bezogen auf den Nickelschmelzpunkt $1452^{\circ}$ ) und etwa $90 \%$ Mangan gefunden.

Von dieser Konzentration an liefern die Abkühlungslinien zwei besondere Punkte bei Temperaturen, welche merklich konstant bleiben mit wachsendem Mangangehalt; dies deutet auf die Gegenwart zweier flüssiger Schichten.

Die obere Horizontale entspricht der Abscheidung primärer Mangankristalle bei der invarianten 'Temperatur von $1180^{\circ}$, sodann der Spaltung der oberen manganreichen Schicht bei dieser Temperatur nach der Reaktion:

Flüssigkeit $D \leftrightarrows$ Primärkristalle von Mangan + Flüssigkeit $c^{\prime}$. 
Wenn die Temperatur sich dann weiter erniedrigt, ändert sich die Zusammensetzung der Flüssigkeit entlang der Kurve $e c^{\prime}$, welche experimentell nicht bestimmt werden konnte wegen des sehr geringen thermischen Effektes.

Der untere Punkt entspricht der Kristallisation der festen Lösung $\boldsymbol{c}$ oder der Verbindung, von welcher noch weiter unten die Rede sein wird. Er findet sich genau bei $980^{\circ}$, also $19^{\circ}$ oberhalb des Schmelzpunktes von Silber; diese Zahl wurde erhalten als Mittelwert von mehr als 30 Bestimmungen, deren äußerste sich um nicht mehr als $3-4^{0}$ voneinander entfernen, wie die Tabelle 1 zeigt.

HINDRICHs hat in gleicher Weise, indem er nach und nach den Mangangehalt von 0 bis etwa $5 \%$ vermehrte, zuerst ein schwaches Fallen der Haltepunkte und dann ein schwaches Ansteigen bemerkt, welches sich in der Tat nach Hindrichs innerhalb der Fehlergrenzen bewegt.

In dem Maße, wie der Gehalt an Mangan sich vermindert, ändern sich die Zeiten der Kristallisation, welche den beiden thermischen Effekten entsprechen, im umgekehrten Sinne bis zur Konzentration von $31 \%$ Mangan, und jenseits dieser Konzentration verschwindet der obere Punkt. Von hier an zeigt die Abkühlungskurve nur noch den unteren Punkt, und zwar immer bei $980^{\circ}$ bis zu einem Gehalt von $21 \%$ Mangan, worauf dann dessen Temperatur sich für jede Legierung entsprechend der Abnahme des Mangangehaltes bis $955^{\circ}$ erniedrigt, wo die Konzentration an Mangan dann noch $4 \%$ beträgt; hierauf steigt die Temperatur wieder bis auf $961^{\circ}$, wo der Schmelzpunkt des reinen Silbers liegt.

Die Temperatur, bei welcher im Diagramm die Horizontale $C F^{\prime}$ gezeichnet ist, $-19^{\circ}$ höher als der Schmelzpunkt des Silbers für Konzentrationen oberbalb $21 \%$ Mangan - würde auf die Gegenwart einer Verbindung mit etwa $21 \%$ Mangan binweisen, deren Schmelzpunkt genau $980^{\circ}$ ist; wäbrend die Abwesenheit eines zweiten Haltepunktes auf den Abkühlungskurven von 0-31\% Mangan, sowie einer eutektischen Horizontalen vielmehr Anzeichen für eine kontinuierliche Reihe von Mischkristallen wären mit einem bis $21 \%$ wachsenden Mangangehalt, wo Sättigung an Mangan vorhanden ist. Diese Mischkristalle würden entlang der Linie $A B C$ kristallisieren, und zwar lägen die Punkte für den Beginn und das Ende der Kristallisation nahe beieinander, daß sie praktisch nicht voneinander unterschieden werden können.

Aber die Tatsache, daB der Punkt $C$ sich in der Nähe der Zu- 
sammensetzung $\mathrm{MnAg}_{2}$, entsprechend $20 \%$ Mangan befindet, spricht für die Tatsache, daB diese Verbindung existiert, und dab sie selbst mit dem Silber eine kontinuierliche Reibe von Mischkristallen bildet, deren Gleichgwichtskurve dargestellt wird durch die Linie $A B C$, die zwischen $A$ und $C$ ein schwaches Minimum besitzt.

Die mikroskopische Prüfung der polierten Oberflächen bestätigt diese Anschauung.

Die Reguli mit geringen Mangangehalten erscheinen in der Tat unter dem Mikroskop homogen; Chlorwasserstoffsäure wirkt auf sie nicht ein, und es ist nicht möglich, das Mangan darin zum Vorschein zu bringen. Beim Ätzen mit Salpetersäure zeigen sie eine regelmäBig zerfressene Oberfläche wie reines Silber. Das Verhalten bleibt so, bis zu einem Gehalt von 20\% Mangan; von dort an beginnt dies Element zu erscheinen. Die Legierung Nr. 28 z. B. mit $18.89 \%$ Mangan zeigt nach Einwirkung von Chlorwasserstoffsäure eine saubere und homogene Fläche, auf welcher man nur einige etwas angegriffene Punkte unterscheidet, sowie 2-3 Tropfen Mangan, welches nicht gelöst ist. Im Gegensatz dazu entsteht bei der Legierung Nr. 6 mit $25 \%$ Mangan durch Chlorwasserstoffsäure ein schwaches Aufschäumen, und unter dem Mikroskop bemerkt man besonders am oberen Teile kleine Mengen von Mangan in Form baumartiger Verzweigungen, von Büscheln oder von Tropfen.

Die Menge des Mangans, die man so nachweisen kann, vermehrt sich mit der Konzentration, wobei eine ausgesprochene Neigung vorhanden ist, sich im oberen Teile der Schmelzen anzuhäufen, bis sich schlieBlich eine deutliche Trennung in zwei Schichten erkennen läbt; dies tritt bereits auf bei einem Mangangehalt ron $35 \%$.

Von dieser Konzentration an sind die beiden Schichten deutlich unterschieden.

Die obere Schicht, welche reich an Mangan ist, wird durch Säuren heftig angegriffen, selbst wenn diese sehr verdünnt sind, und es treten dabei nicht angegriffene Stellen hervor, die mehr oder weniger abgerundet, gezähnt und schwach gelb gefärbt sind. Sie bestehen aus der Verbindung $\mathrm{MnAg}_{2}$.

Die untere silberreiche Schicht schäumt noch mit verdünnter Chlorwasserstoffsäure schwach auf und läBt dann auf dem nicht angegriffenen Grunde der Verbindung oder der festen Lösung Dendriten von Mangan erkennen.

Die Legierungen mit mehr als $95 \%$ Mangan sind so brüchig, daß sie nicht poliert und unter dem Mikroskop geprüft werden können. 
Die chemische Untersuchung bestätigt die angegebenen Tatsachen und erlaubt, in noch genauerer Weise die Zusammensetzung der Verbindung oder des Bestandteiles $\alpha$ anzugeben.

Wenn man versucht, durch längere Einwirkung von Säure auf die Metallschmelzen die nachgewiesenen Bestandteile zu trennen, so erkennt man nach beendigter Einwirkung, daB der Rückstand eine sehr beträchtliche Menge Mangan zurückhält, die man ihm nicht entziehen kann, selbst wenn man ihn mehrfach zerreibt und mit Säuren behandelt. Die Menge des so gebundenen Mangans beträgt etwa $20 \%$ und bisweilen noch 2 oder $3 \%$ mehr, wenn das erhaltene Pulver nicht sehr fein war.

Eine Legierung von $90 \%$ Mangan z. B. hinterließ nach sehr feinem Pulvern und Behandlung mit Chlorwasserstoffsäure einen metallischen Rückstand, der aus glänzenden Blättchen bestand, die bei der Analyse nach mehrfacher Behandlung mit Säuren und mehrfacher Zerkleinerung $78.55 \% \mathrm{Ag}$ and $21.40 \% \mathrm{Mn}$ lieferten, wobei keine merklichen Mengen von Silber in die auflösende Säure hineingingen.

Um diese Rückstände zu lösen, muß man Salpetersäure oder konzentrierte Schwefelsäure anwenden.

Um zu sehen, ob durch gemäBigte Säurewirkung die Zusammen. setzung der Verbindung konstant bleibt, behandelte man $2.8 \mathrm{~g}$ des Pulvers mit Salpetersäure von mäBiger Konzentration in der Wärme; nach einer halben Stunde war das Gewicht nur noch $1.2 \mathrm{~g}$, was einem Verlust von mehr als $50 \%$ entspricht. Die Analyse dieses letzten Rückstandes ergab noch einen Gehalt von 21.05\% Mangan und $78.41 \%$ Silber.

Die früheren Versuche mit aluminothermisch dargestellten Legierungen hatten uns Rückstände geliefert, die etwas weniger Mangan enthielten, nämlich etwa $20 \%$, woraus wir auf die Formel $\mathrm{MnAg}_{2}$ geschlossen hatten.

Die Untersuchung der elektromotorischen Kräfte bringt weitere Stützen für die Existenz dieser Verbindung $\mathrm{MnAg}_{2}$.

Diese Untersuchung ist ausgeführt worden nach dem Verfahren von Puschin, in der von Vigouroux vorgeschlagenen Abänderung. ${ }^{1}$ Die gefundenen Zahlen sind in Tabelle 2 zusammengestellt nach welcher die Kurve (Fig. 2) konstruiert ist.

1 Contribution a l'étude du sytème Nickel-cuivre. Bull. soc. chim. de France [4] 7, 191. 


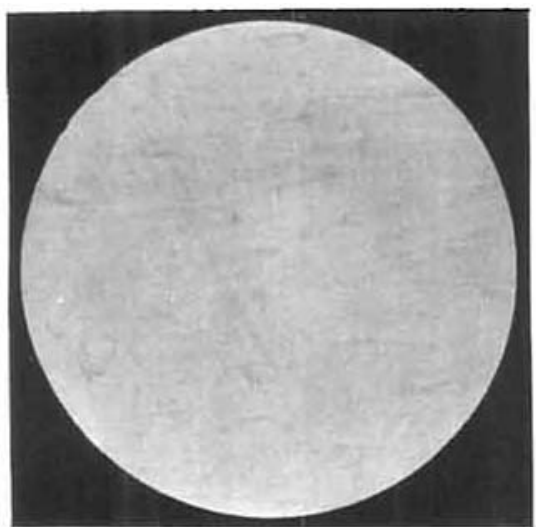

Fig. 1. Mn-Ag-Legierungen Nir. 27 mit $17.35 \%$ Ag. Mit HCl gestat. Vergr. $300 \times$.

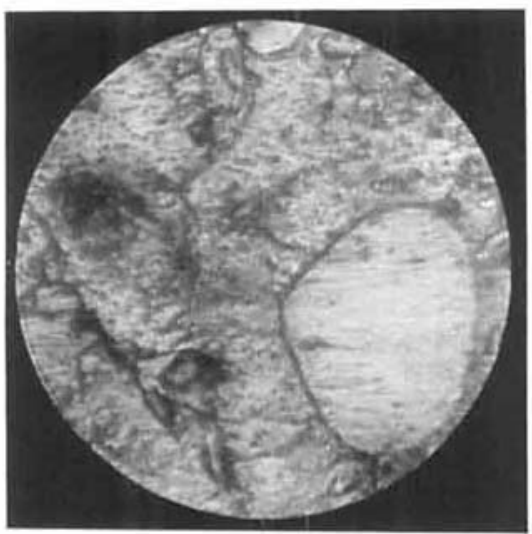

Fig. 3. MIn-Ag-Legierungen Nr. 36 mit $60 \%$ Mn. Obere Schicht mit $90 \% \mathrm{Mn}$.

Vergr. $300 x$.

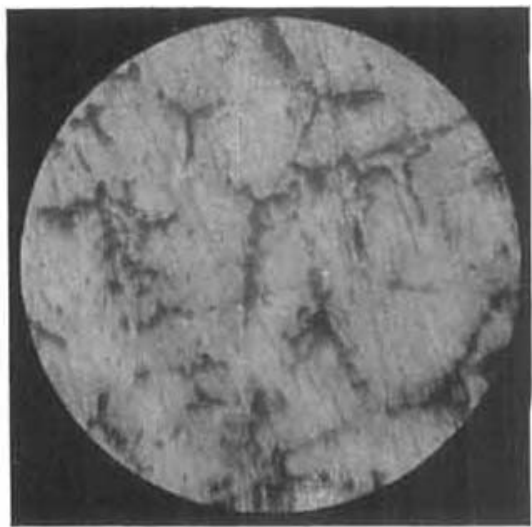

Fig. 2. Mu-Ag-Legierungen Nr. 6 mit $25 \%$ Hn. Geützt m. Essigsliure. Vergr. $100 \times$

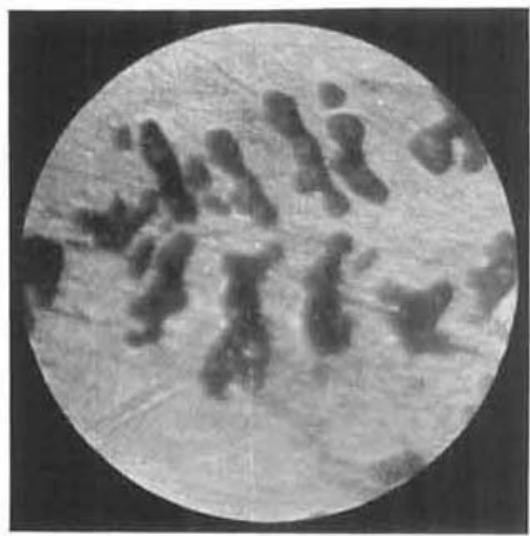

Fig. 4. Mu-Ag-Legierungen Nr. 38 mit $60 \% \mathrm{Mn}$. Geätat mit verd. Salzshure. Untere Schicht mit $27 \%$ hIn. Vergr. $300 \times$.

G. Amtrant.

Verlag von Leopold Voss in Leipzig und Hamburg. 
Tabelle 2.

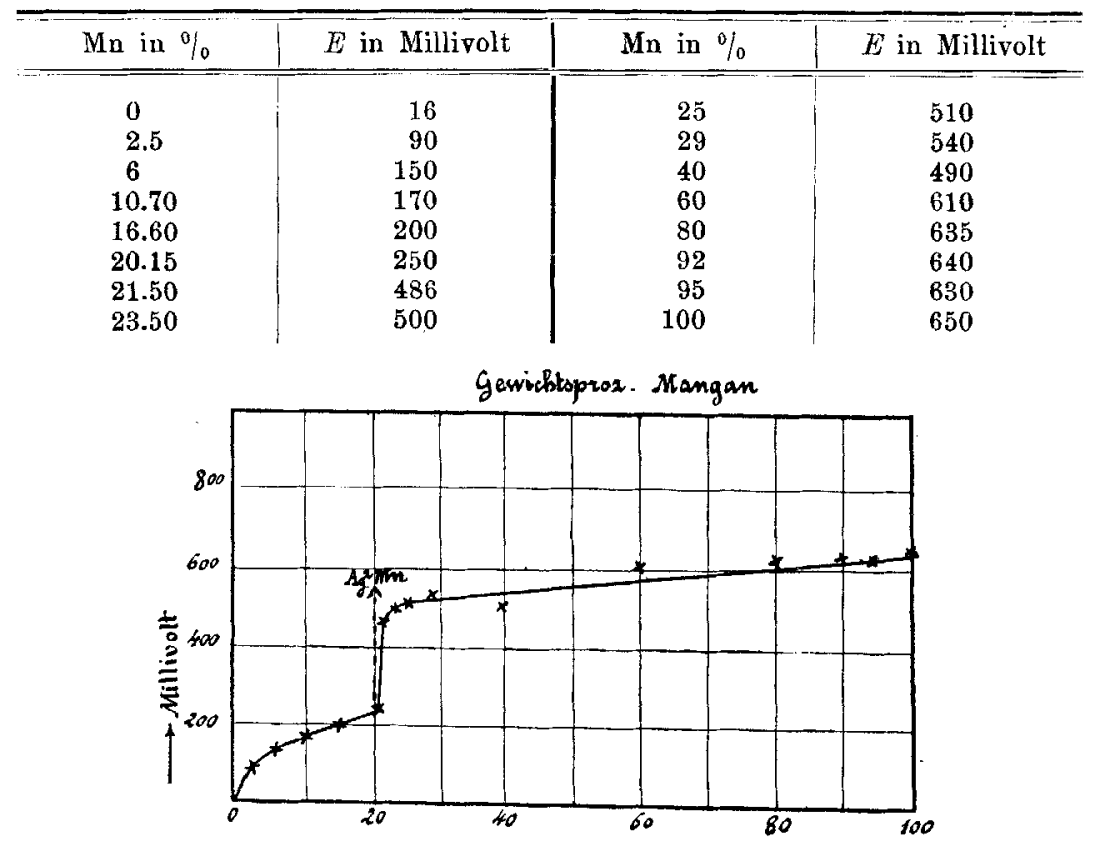

Fig. 2.

Elekromotorische Kräfte der Mn-Ag-Legierungen.

Die elektromotorischen Kräfte sind ausgedrückt in Millivolt und bezogen auf eine mit Mangandioxyd depolarisierte Silberelektrode in einer 0.1-norm. Mangansulfatlösung.

Der starke Abfall der elektromotorischen Kräfte zwischen den Konzentrationen von $23 \%$ und 20\% Mangan deutet auf die Gegenwart einer Verbindung, während gleichzeitig ihre allmähliche Abnahme von 20-0\% Mangan auf die Bildung von Mischlristallen zwischen dieser Verbindung und Silber hinweist.

Die verschiedenen Untersuchungsmethoden führen also übereinstimmend zu dem SchluB, daB Mangan und Silber eine Verbindung $\mathrm{MnAg}_{2}$ mit $20 \%$ Mangan bilden können, die in Chlorwasserstoffsäure unlöslich ist.

Diese Verbindung gibt mit Silber eine kontinuierliche Reihe von Mischkristallen, und die Grenze der Mischbarkeit im fiüssigen Zustand zwischen Mangan und dieser Verbindung liegt bei etwa 30\% Mangan.

Bordeaux, Laboratoire de Chimie industrielle de l'université.

Bei der Redaktion eingegangen am 7. Mai 1913. 